Large-area integrated triboelectric sensor array for wireless static and dynamic pressure detection and mapping

Hai Lu Wang, Shuang Yang Kuang, Hua Yang Li, Zhong Lin Wang, Guang Zhu

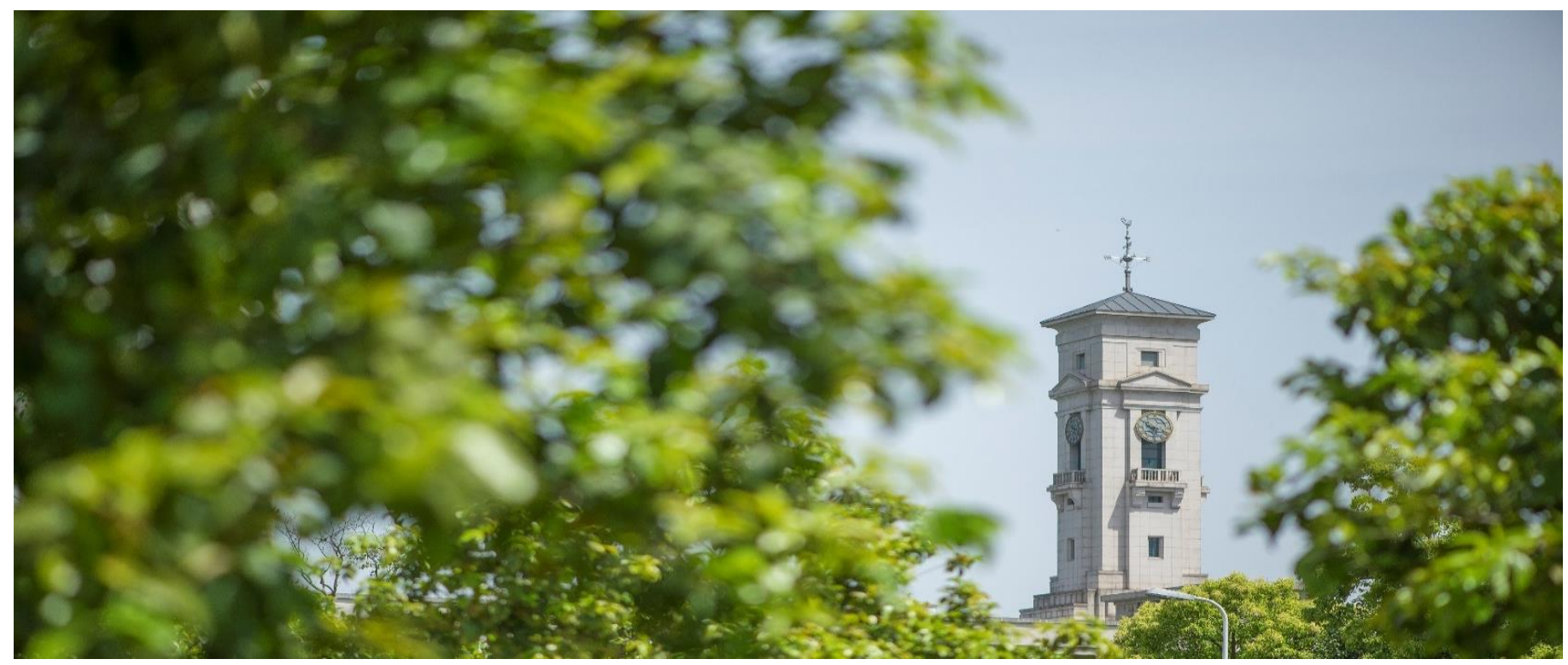


University of Nottingham Ningbo China, 199 Taikang East Road, Ningbo, 315100, Zhejiang, China.

First published 2019

This work is made available under the terms of the Creative Commons Attribution 4.0 International License:

http://creativecommons.org/licenses/by/4.0

The work is licenced to the University of Nottingham Ningbo China under the Global University Publication Licence:

https://www.nottingham.edu.cn/en/library/documents/researchsupport/global-university-publications-licence.pdf 


\title{
Large-Area Integrated Triboelectric Sensor Array for Wireless Static and Dynamic Pressure Detection and Mapping
}

Hai Lu Wang, Shuang Yang Kuang, Hua Yang Li, Zhong Lin Wang, Guang Zhu*

\author{
H. L. Wang, Z. L. Wang, Prof. G. Zhu
}

CAS Center for Excellence in Nanoscience, Beijing Key Laboratory of Micro-nano Energy and Sensor, Beijing Institute of Nanoenergy and Nanosystems, Chinese Academy of Sciences, Beijing 100083, P. R. China

E-mail: zhuguang@binn.cas.cn

H. L. Wang, Prof. Z. L. Wang, Prof. G. Zhu

School of Nanoscience and Technology, University of Chinese Academy of Sciences, Beijing 100048, P. R. China

S. Y. Kuang

MOE Key Laboratory of Fundamental Physical Quantities Measurement \& Hubei,

Key Laboratory of Gravitation and Quantum Physics, PGMF and School of Physics, Huazhong University of Science and Technology, Wuhan 430074, P. R. China.

New Materials Institute, Department of Mechanical, Materials and Manufacturing Engineering, University of Nottingham Ningbo China, Ningbo 315100, P. R. China Prof. Z. L. Wang, Prof. G. Zhu

Center on Nanoenergy Research, School of Physical Science and Technology, Guangxi University, Nanning 530004, P. R. China

H.Y. Li, Prof. G. Zhu

Prof. Z. L. Wang

School of Materials Science and Engineering, Georgia Institute of Technology, Atlanta GA 30332, USA

Keywords: triboelectric nanogenerator, field effect transistor, large area, pressure mapping, motion monitoring. 


\section{Abstract}

Large-area flexible pressure sensors are of paramount importance for various future applications, such as electronic skin, human-machine interfacing, and healthmonitoring devices. Here, we report a self-powered and large-area integrated triboelectric sensor array (ITSA) based on coupling a triboelectric sensor array (TSA) and an array chip of CD4066 through a traditional connection way. Enabled by a simple and cost-effective fabrication process, the size of the ITSA can be scaled up to $38 \times 38$ $\mathrm{cm}^{2}$. In addition, unlike the proposed triboelectric sensors array before which can only react to the dynamic interaction, this ITSA is able to detect static and dynamic pressure. Moreover, through integrating the ITSA with a signal processing circuit, a complete wireless sensing system is present. Diverse applications of the system are demonstrated in details, including detecting pressure, identifying position, tracking trajectory and recognizing the profile of external contact objects. Thus, the ITSA in this work opens a new route in the direction of large-area, self-powered, and wireless triboelectric sensing system.

\section{Introduction}

Flexible pressure sensors have drawn increasing attentions in the last decade by virtue of their enormous potential for uses in electronic skin, ${ }^{[1-3]}$ human-machine interfacing, ${ }^{[4-7]}$ health-monitoring devices ${ }^{[1-2,8-9]}$ and human-motion detection ${ }^{[2,9-10]}$ etc. In terms of practical applications, it is crucial for the pressure sensors to possess attributes of easy fabrication, high sensitivity, excellent durability, low power consumption and a possibility to be scaled up in large area. Although pressure sensors 
based on piezoresistive ${ }^{[6,11-12]}$ and capacitive $e^{[13-15]}$ transducing mechanisms have been previously reported with decent sensitivity and reliability, the dependence on a constant external power supply is still an issue especially when a large-area sensor array is to be made. Recently, a new-type self-powered pressure sensor based on the triboelectric nanogenerator (TENG) have been developed. ${ }^{[16-20]}$ This kind of sensor could transform external mechanical signals directly into electric signals in the absence of external power supplies, exactly resolving the problem of energy consumption for the sensing unit. ${ }^{[21]}$ Nevertheless, some limitations of the triboelectric pressure sensors still need to be overcome. The triboelectric sensors could produce electric signals only when they were in dynamic interaction with external stimuli. ${ }^{[22-23]}$ The generated electric signals were instantaneous and transient, and their amplitude were correlated to how fast the stimuli were exerted. Both the pressure and the velocity of the stimuli could influence the electric signal's amplitude in a coupled way, it posed a major challenge to separately and precisely quantify some important static parameters such as pressure and displacement. To address the issue above, some efforts have been made to develop triboelectric sensors coupled with field effect transistors (FETs). ${ }^{[24-27]}$. This hybrid pressure sensor requires no external gate voltage, the output voltage of the TENG acts as the gate bias to modulate the drain-source current $\left(I_{\mathrm{DS}}\right)$ across the FET. Thus, the transient electric signals generated by the TENG can be converted to stable $I_{\mathrm{DS}}$ signals. ${ }^{[26]}$ In addition, the output voltage of the TENG is only associated with the pressure level of the external stimuli and has nothing to do with the velocity. Therefore, this hybrid device can effectively decouple the joint influence of pressure and velocity 
of the external stimuli. For instance, U. Khan.et. al ${ }^{[25]}$ reported an array of tribotronic touch sensors constructed by coplanar coupling of a TENG and a graphene FET, and demonstrated the application of mapping multi-points touch and tracking a moving ball. And Z. W. Yang. et. al ${ }^{[28]}$ achieved touch map reconstruction, motion detecting, and trajectory tracking by using a tribotronic transistor array, which was incorporated by TENGs and FETs. However, most of the proposed devices were constructed through complicated and delicate fabrication process, such as UV lithography technique or electron beam evaporation, ${ }^{[24-25,27]}$ etc. Additionally, materials that can be utilized are also confined to the semiconductor materials. ${ }^{[26-27,29]}$ These key issues make the construction and scale-up for the FET-based triboelectric sensors remain challenging. Therefore, it is highly urgent to develop a type of convenient-prepared and large-area TENG-FET hybrid pressure sensor that can measure static and dynamic pressure for pressure and positioning mapping.

Herein, we demonstrate a self-powered integrated triboelectric sensor array (ITSA), which is constructed by a universal combination of a triboelectric sensor array (TSA) with an array chip of CD4066. Enabled by a simple and convenient fabrication process, the size of the ITSA can be scaled up to $38 \times 38 \mathrm{~cm}^{2}$, which is much larger than the previous reported TENG-FET hybrid sensor arrays. ${ }^{[24-25,30]}$ Without any external gate voltage, the ITSA can achieve a stable current signal response under extremely low pressure $(0.24 \mathrm{kPa})$ no matter how fast the external stimuli is. In addition, through integrating the ITSA with a signal processing circuit, a prototype of wireless sensing system is demonstrated. It is well capable of detecting pressure, identifying 
position, tracking trajectory and recognizing the profile of external contact objects in a wireless visualized way. The ITSA exhibits distinct advantages in self power, low cost, sustainability and scalability, indicating that it has enormous potential in applications for human-motion monitoring system, security monitoring system, and human-machine interface platforms.

\section{Results and discussion}

The break-down structure of the TSA is illustrated in Figure 1a and b. One single sensor unit consists of the following components: a top substrate layer made of silicone, a top electrode layer made of woven conductive fabric, a layer of elastic balls with the diameter of $5 \mathrm{~mm}$, a electrification layer made of polytetrafluoroethylene (PTFE) film, a bottom electrode layer made of woven conductive fabric, two insulation layers and a grounded shielding layer. And the woven conductive fabric shows great excellent robustness and stability under bending state, which enables the flexibility and bendability, as shown in Figure S5 (Supporting Information). All of them were constructed on the bottom substrate layer made of silicone sequentially. The two substrate layers cover all the components. The top electrode layer and the PTFE layer are separated by the elastic balls between them, while the bottom electrode layer is laid under the electrification layer. The grounded shielding layer here is utilized to restrain the crosstalk, it has two insulation layers on both sides to avoid short circuits. The shielding layer can effectively eliminate electrostatic induction between electrode wires in vertical direction, and this will be discussed in detail later. A row structure of the TSA is demonstrated in Figure 1b. The top electrode layer is lined up by eight units with 
solid squares, while the bottom electrode layer is formed from eight independent square units. The units between the two electrodes have up-down and one-to-one correspondence. The scanning electron microscope (SEM) image of the top electrode is shown in Figure 1c, which is essentially woven fabric deposited with metal. In order to improve the triboelectric charge density after contacts with the top electrode, the PTFE film was treated with plasma etching, ${ }^{[16,31]}$ and the SEM image in Figure 1d indicates the nanorods on the PTFE film. The increased output voltage of the treated PTFE film was shown in Figure S6 (Supporting Information). An as-fabricated TSA is sketched in Figure 1e, which shows great flexibility and bendability. As demonstrated in Figure 1f, the TSA with an area of $38 \times 38 \mathrm{~cm}^{2}$ is large enough to accommodate an adult's foot, indicating the possible applications of the TSA in human motion monitoring. This simple fabrication process as well as the cost-effective materials make the TSA compatible to be fabricated in large scale.

The basic sensing mechanism of the TSA can be explained by a cross-sectional schematic diagram sketched in Figure 2a. It relies on the coupling of contact electrification and electrostatic induction between the PTFE film and the top electrode $^{[21]}$. In the original state, a separation between the PTFE film and the top electrode is maintained by the elastic balls between them, as shown in Figure 2a (i). When an external force is applied on the TSA, the PTFE film and the top electrode are brought into contact. According to the triboelectric series ${ }^{[21]}$, PTFE is much more triboelectrically negative than the electrode, so the electrons are injected from the top electrode to PTFE surface. As a result, triboelectric charges of opposite signs are 
generated at the contact surfaces, with negative ones on the PTFE side and positive ones on the top electrode side, as shown in Figure 2a (ii). Since the opposite charges coincide at the same plane, there is almost no electric potential difference between the two electrodes. Once the external force is withdrawn, the top electrode tends to revert back to its original position due to the resilience of silicone and then the electric potential difference is established between the two electrodes, as shown in Figure 2a (ii)-(iv). At open-circuit condition, the open-circuit voltage $V_{\mathrm{OC}}$ across the electrode at the separation state is given by: ${ }^{[32]}$

$$
V_{\mathrm{OC}}=-\frac{\sigma d}{\varepsilon_{0}}
$$

where $\sigma$ is the triboelectric charge density of PTFE film, $d$ is the separation distance between the top electrode layer and PTFE film, $\varepsilon_{0}$ is dielectric constant of vacuum. The $V_{\text {oc }}$ reaches the maximum value when the top electrode fully reverts to the original position, as shown in Figure 2a (iv). For better characterizing the response capability of the TSA, we define output voltage as the difference of the $V_{\text {oc }}$ with respect to that at the maximum-separation state (Figure 2a (iv)). Therefore, the output voltage value always shows positive since it has a zero-baseline corresponding to separation state. Under different external pressure, the corresponding output voltage of the sensor unit with the size of $4 \times 4 \mathrm{~cm}^{2}$ is sketched in Figure $2 \mathrm{~b}$. The constant external pressure was applied by a mechanical linear motor and monitored by a dynamometer. The output voltage increases to the maximum value with the applied pressure increasing from 0.1 $\mathrm{kPa}$ to $37.5 \mathrm{kPa}$, as shown in Figure 2c. The curve exhibits three distinct regions based on pressure sensitivity. In low pressure region $(0.1 \mathrm{kPa} \sim 0.7 \mathrm{kPa})$, the pressure 
sensitivity is $53.7 \mathrm{mV} / \mathrm{Pa}$ (linearity $\left.\mathrm{R}^{2}=0.996\right)$. While in medium pressure region $(2.5$ $\mathrm{kPa} \sim 21.5 \mathrm{kPa}$ ), the pressure sensitivity reaches $5.3 \mathrm{mV} / \mathrm{Pa}$ (linearity $\mathrm{R}^{2}=0.991$ ). And in high pressure region $25.5 \mathrm{kPa} \sim 37.5 \mathrm{kPa}$, the pressure sensitivity decreases to 1.2 $\mathrm{mV} / \mathrm{Pa}$ (linearity $\mathrm{R}^{2}=0.980$ ). These different sensitivities can be explained by the variations of separate distance and contact areas based on previous literature. ${ }^{[16,33]}$ In low pressure region, the output voltage variation is primarily attribute to the different separation distance between the PTFE film and top electrode, as estimated in Equation 1. While in medium and high pressure regions, the output voltage change relies on the increasing contact areas. It is suggested that the different sensitivity in medium and high pressure regions is caused by the different change rates of efficient contact area, which is discussed detailedly in Figure S1 (Supporting Information). Moreover, the durability test was also conducted to confirm the stability of the TSA for more than 30000 cycles, as shown in Figure 2d. Due to the robust structure and durable materials, the TSA shows excellent sustainability.

In practical measurements, external objects contact with the TSA dynamically and arbitrarily, which inevitably causes measurable voltage between the electrode lines among adjacent sensor units. Therefore, the issue of crosstalk signal in the TSA should be solved firstly. Here, we utilized a grounded shielding layer to successfully suppress the crosstalk, it proved to be effective in eliminating the cross talk according to previous work. ${ }^{[22-23]}$ The result is shown in Figure 2e, eight sensor units that come from one column of the TSA are labeled as $1,2 \ldots 8$, respectively. The output voltage of the unit 4 is measured when the external pressure applied on these eight units successively. 
Without the shielding layer, comparable output voltage values of unit 4 are detected whichever unit is triggered, as shown in Figure S2 (Supporting Information). In comparison with the presence of shielding layer, the voltage reaches more than $16 \mathrm{~V}$ when unit 4 is triggered, while the maximum voltage is less than $2 \mathrm{~V}$ when the other seven units are triggered, as shown in Figure 2e. This significant difference is attributed to the grounded shielding layer, which effectively eliminates the induced charges among electrode lines. Furthermore, the output voltage signals of the TSA were measured when a model with shape of " $\mathrm{N}$ " was pressed on the top surface. And an obvious contrast between the mapping of the pressed and unpressed units was observed in Figure 2f, from which the shape of "N" was distinctly imaged. This expectant result validates that the TSA has good practicability for position recognition and pressure mapping.

The output electric signals produced by the TSA can be easily picked up by external electric circuits. Therefore, we incorporated a sensor unit in the TSA with a MOSFET. And Figure 3a shows the circuit diagram of the traditional combination between them, in which the gate voltage of the MOSFET is modulated by the output voltage of the sensor unit. When the unit was triggered, external applied pressure, output voltage values of the sensor unit and drain-source current $\left(I_{\mathrm{DS}}\right)$ of the MOSFET are measured synchronously, which are shown in Figure 3b. As a result, the MOSFET is switched off when external pressure is below $0.10 \mathrm{kPa}$, which does not generate a current across the source and drain. While when the pressure is between $0.10 \mathrm{kPa}$ and $0.24 \mathrm{kPa}$, the MOSFET works as a resistor and the $I_{\mathrm{DS}}$ increases with the increasing 
external pressure. Finally, when the pressure is beyond $0.24 \mathrm{kPa}$, the MOSFET is turned on, giving rise to a stable $I_{\mathrm{DS}}$ value of $0.5 \mathrm{~mA}$. It therefore can function as a switch circuit by virtue of the change of $I_{\mathrm{DS}}$. As illustrated in Figure $3 \mathrm{c}$, the switch circuit can be turn on under extremely low pressure $(0.24 \mathrm{kPa})$, and produces sustainable current signals of $0.5 \mathrm{~mA}$. This enables the capability for static pressure detection, unlike the TSA, which can only produce pulsed signals during the process of contact/separation, as shown in Figure S7 (Supporting Information). For large area mapping and human positioning application, an array chip of CD4066 that contains 64 single FET-based analogue switches was integrated with the TSA to build up a self-powered and flexible ITSA. This system can be thought of an eight-by-eight matrix keyboard, in which the FET in each analogue switch of CD4066 array chip is controlled by a corresponding sensor unit in the TSA. The equivalent circuit diagram with eight input lines I1 to I8 and eight output lines $\mathrm{O} 1$ to $\mathrm{O} 8$ is demonstrated in Figure $3 \mathrm{~d}$ (left part). The detailed working principle of CD4066 is present in Figure S3 (Supporting Information). The junction circuit between entire 64 sensor units in the TSA and the CD4066 array chip is schematically illustrated in Figure S4 (Supporting Information).

Moreover, in order to detect pressure in a visualized way, an ultra-low power consumption microprogrammed control unit (MCU-MSP430) was further integrated into the aforementioned system. This integrated sensor array can realize visually detecting pressure and identifying position. As shown in Figure 3d (right part), the eight input signal lines I1 to I8 in CD4066 array chip are connected to pin P3.0, P3.1 ... P3.7 on $\mathrm{MCU}$, and eight output signal lines $\mathrm{O} 1$ to $\mathrm{O} 8$ are connected to pin $\mathrm{P} 2.0, \mathrm{P} 2.1 \ldots$ 
P2.7, respectively. As for pin P2.0 to P2.7, they were set high level primordially, while as for pin P3.0 to P3.7, they were circularly set a low level signal lasts for $0.2 \mathrm{~ms}$ one after the other, as shown in Figure 3e. Here, for better describing the position of sensor units in the ITSA, the sensor unit was named as $\mathrm{S}_{\mathrm{XY}}$ who locates at intersecting coordinate of Row X and Column Y, respectively. The work principle for positioning of this system is demonstrated in detail in Figure 3f. In single point test (left part of Figure 3f), when sensor unit $\mathrm{S}_{41}$ is pressed by a finger, input line I1 is electrically connected with output line $\mathrm{O} 4$. As a result, one low level signal which is identical to that in pin P3.0 can be detected at pin P2.3. In multi-point tests, it was conducted in two points and four points test, as demonstrated in Figure $3 \mathrm{f}$ (medium and right parts). When sensor $\mathrm{S}_{41}$ and $\mathrm{S}_{43}$ are pressed simultaneously, resulting in the electric connection of input line I1 and output line O4, input line I3 and output line O4, respectively. Therefore, two low level signals with time interval of $0.2 \mathrm{~ms}$ at pin P2.3 can be detected, which is corresponding to low level signal in pin P3.0 and P3.2. Spontaneously, when pressure were applied on $\mathrm{S}_{41}, \mathrm{~S}_{43}, \mathrm{~S}_{44}$ and $\mathrm{S}_{45}$ simultaneously, input line I1, I3, I4 and I5 are electrically connected with output line O4, respectively. Consequently, four low level signals at pin P2.3 corresponding to that in P3.0, P3.2, P3.3 and P3.4 can be detected. These signals are processed by MCU and then reveal the position information of triggered sensor units to us. Thus, the low level signals detected at pin P2.Y ( $\mathrm{Y}=0,1 \ldots$ 7) which is corresponding to the signals at P3.X $(X=0,1 \ldots 7)$, can address the row line (row $\mathrm{Y}+1)$ and column line (column $\mathrm{X}+1)$ of the triggered unit.

Finally, a high-performance sub-1-GHz Radio Frequency (RF) transceiver is 
integrated with the above system to achieve wireless sensing, as shown in Figure 4. Electric signal that carries position information is modulated to a $433 \mathrm{MHz}$ carrier wave, which is emitted by an LC oscillation circuit to outer space. A receiver far away receives the carrier wave and demodulates the information from the carrier wave, this process is graphically illustrated in Figure 4a. As a result, once an object is loaded on the sensor matrix, its information including pressure and position as well as spatial mapping and movement tracking can be recognized by the central processing unit (CPU). The practical exhibition can be seen in Supporting Movies. In the foot stepping experiments which is shown in Figure $4 \mathrm{~b}$ and $4 \mathrm{c}$, the position and contacting contour of the feet can be synchronously displayed on the screen of the computer far away, in which the evident difference between the man's and the woman's shoes contour can also be observed. Thus, the ITSA can function as a "smart carpet". The ITSA reveals a resolution of $8 \times 8$, and the resolution directly depends on the number of sensor units per unit area. It is believed that a high-resolution area-scalable "smart carpet" is attainable, since there is a lot of room for improvement in resolution by decreasing the size of sensor unit without reducing the output, which can be achieved by means of modifying and tailoring the triboelectrification materials. ${ }^{[31,34-35]}$ For further applications of the ITSA, the capability for spatial pressure mapping is exhibited in Figure 4d-f. When a group of counterweights with different weights range from $20 \mathrm{~g}$ to $500 \mathrm{~g}$ are loaded on the ITSA (Figure 4d). The triggered sensor units were identified by measuring the output voltage as shown in Figure 4e, in which the height of each bar corresponds to the output voltage of the sensor units. And Figure 4f shows the reconstructed pressure 
mapping, in which the color contrast corresponds to the calibrated actual pressure. All things considered, the large area ITSA developed here enables tremendous potential applications in human-motion monitoring system, security system and human-machine interface.

\section{Conclusion}

In summary, we report a large-area $\left(38 \times 38 \mathrm{~cm}^{2}\right)$ and self-powered integrated triboelectric sensor array (ITSA) based on coupling of a triboelectric sensor array (TSA) and an array chip of CD4066. This flexible and durable ITSA can sense external static and dynamic pressure through the change of drain-source current $\left(I_{\mathrm{DS}}\right)$, and the switch circuit can be turn on under extremely low pressure $(0.24 \mathrm{kPa})$. Further integrated with the MCU-MSP430, the system that is capable of precisely identifying position and tracking trajectory was proposed. Additionally, a complete wireless sensing system is developed by further incorporation with the sub-1-GHz RF transceiver, and remote control and monitoring has been achieved. The ITSA demonstrates the application in positioning, trajectory tracking and identifying of approximate shape of contact objects. Considering that the ITSA feathers advances in cost and scalability, we believe it presents feasible applications in human-motion monitoring systems, electric skins and human-machine interface systems.

\section{Supporting Information}

Supporting Information is available from the Wiley Online Library or from the author.

\section{Acknowledgements}

This research was supported by the National Key R\&D Project from Ministry of Science and Technology, China (Grant No. 2016YFA0202701\&2016YFA0202703), 
National Science Foundation of China (Grant No. 51572030), Beijing Natural Science Foundation (Grant No. 2162047). Hai Lu Wang, Shuang Yang Kuang and Hua Yang Li contributed equally to this work.

\section{References}

[1] G. Schwartz, B. C. K. Tee, J. G. Mei, A. L. Appleton, D. H. Kim, H. L. Wang, Z. N. Bao, Nature Communications 2013, 4,

[2] Z. J. Zhu, R. Y. Li, T. R. Pan, Advanced Materials 2018, 30,

[3] L. Nela, J. S. Tang, Q. Cao, G. Tulevski, S. J. Han, Nano Letters 2018, 18, 2054.

[4] T. Chen, Q. F. Shi, M. L. Zhu, T. Y. Y. He, L. N. Sun, L. Yang, C. Lee, Acs Nano 2018, 12, 11561.

[5] X. Q. Liao, W. T. Song, X. Y. Zhang, H. Huang, Y. T. Wang, Y. J. Zheng, Journal of Materials Chemistry C 2018, 6, 12841.

[6] J. Lee, S. Shin, S. Lee, J. Song, S. Kang, H. Han, S. Kim, S. Kim, J. Seo, D. Kim, T. Lee, Acs Nano 2018, 12, 9634.

[7] H. Chang, S. Kim, S. Jin, S. W. Lee, G. T. Yang, K. Y. Lee, H. Yi, Acs Applied Materials \& Interfaces 2018, 10, 1067.

[8] H. Ouyang, J. J. Tian, G. L. Sun, Y. Zou, Z. Liu, H. Li, L. M. Zhao, B. J. Shi, Y. B. Fan, Y. F. Fan, Z. L. Wang, Z. Li, Advanced Materials 2017, 29,

[9] M. M. Liu, X. Pu, C. Y. Jiang, T. Liu, X. Huang, L. B. Chen, C. H. Du, J. M. Sun, W. G. Hu, Z. L. Wang, Advanced Materials 2017, 29,

[10] G. Ge, Y. C. Cai, Q. C. Dong, Y. Z. Zhang, J. J. Shao, W. Huang, X. C. Dong, Nanoscale 2018, 10, 10033. 
[11] W. J. Liu, N. S. Liu, Y. Yue, J. Y. Rao, F. Cheng, J. Su, Z. T. Liu, Y. H. Gao, Small 2018, 14,

[12] Y. N. Ma, Y. Yue, H. Zhang, F. Cheng, W. Q. Zhao, J. Y. Rao, S. J. Luo, J. Wang, X. L. Jiang, Z. T. Liu, N. S. Liu, Y. H. Gao, Acs Nano 2018, 12, 3209.

[13] S. G. Yoon, B. J. Park, S. T. Chang, Acs Applied Materials \& Interfaces 2017, 9,36206 .

[14] R. Nur, N. Matsuhisa, Z. Jiang, M. O. G. Nayeem, T. Yokota, T. Someya, Nano Letters 2018, 18, 5610.

[15] H. Kim, G. Kim, T. Kim, S. Lee, D. Kang, M. S. Hwang, Y. Chae, S. Kang, H. Lee, H. G. Park, W. Shim, Small 2018, 14,

[16] G. Zhu, W. Q. Yang, T. J. Zhang, Q. S. Jing, J. Chen, Y. S. Zhou, P. Bai, Z. L. Wang, Nano Letters 2014, 14, 3208.

[17] M. Ha, S. Lim, S. Cho, Y. Lee, S. Na, C. Baig, H. Ko, Acs Nano 2018, 12, 3964.

[18] P. Bai, G. Zhu, Q. S. Jing, J. Yang, J. Chen, Y. J. Su, J. S. Ma, G. Zhang, Z. L. Wang, Advanced Functional Materials 2014, 24, 5807.

[19] X. D. Wang, H. L. Zhang, L. Dong, X. Han, W. M. Du, J. Y. Zhai, C. F. Pan, Z. L. Wang, Advanced Materials 2016, 28, 2896.

[20] K. Parida, V. Bhavanasi, V. Kumar, R. Bendi, P. S. Lee, Nano Research 2017, 10,3557 .

[21] Z. L. Wang, Acs Nano 2013, 7, 9533. 
[22] X. X. Zhu, X. S. Meng, S. Y. Kuang, X. Di Wang, C. F. Pan, G. Zhu, Z. L. Wang, Nano Energy 2017, 41, 387.

[23] X. X. Zhu, Z. B. Li, X. S. Li, L. Su, X. Y. Wei, S. Y. Kuang, B. W. Su, J. Yang, Z. L. Wang, G. Zhu, Nano Energy 2018, 50, 497.

[24] F. Xue, L. B. Chen, L. F. Wang, Y. K. Pang, J. Chen, C. Zhang, Z. L. Wang, Advanced Functional Materials 2016, 26, 2104.

[25] U. Khan, T. H. Kim, H. Ryu, W. Seung, S. W. Kim, Advanced Materials 2017, 29,

[26] C. Zhang, W. Tang, L. M. Zhang, C. B. Han, Z. L. Wang, Acs Nano 2014, 8, 8702.

[27] G. Y. Gao, B. S. Wan, X. Q. Liu, Q. J. Sun, X. N. Yang, L. F. Wang, C. F. Pan, Z. L. Wang, Advanced Materials 2018, 30,

[28] Z. W. Yang, Y. K. Pang, L. M. Zhang, C. X. Lu, J. Chen, T. Zhou, C. Zhang, Z. L. Wang, Acs Nano 2016, 10, 10912.

[29] C. Zhang, J. Li, C. B. Han, L. M. Zhang, X. Y. Chen, L. D. Wang, G. F. Dong, Z. L. Wang, Advanced Functional Materials 2015, 25, 5625.

[30] Y. K. Pang, J. Li, T. Zhou, Z. W. Yang, J. J. Luo, L. M. Zhang, G. F. Dong, C. Zhang, Z. L. Wang, Nano Energy 2017, 31, 533.

[31] L. M. Zhao, Q. Zheng, H. Ouyang, H. Li, L. Yan, B. J. Shi, Z. Li, Nano Energy 2016, 28, 172.

[32] S. M. Niu, S. H. Wang, L. Lin, Y. Liu, Y. S. Zhou, Y. F. Hu, Z. L. Wang, Energy \& Environmental Science 2013, 6, 3576. 
[33] L. Lin, Y. N. Xie, S. H. Wang, W. Z. Wu, S. M. Niu, X. N. Wen, Z. L. Wang, Acs Nano 2013, 7, 8266.

[34] H. Y. Li, L. Su, S. Y. Kuang, C. F. Pan, G. Zhu, Z. L. Wang, Advanced Functional Materials 2015, 25, 5691.

[35] Z. H. Lin, Y. N. Xie, Y. Yang, S. H. Wang, G. Zhu, Z. L. Wang, Acs Nano $2013,7,4554$.

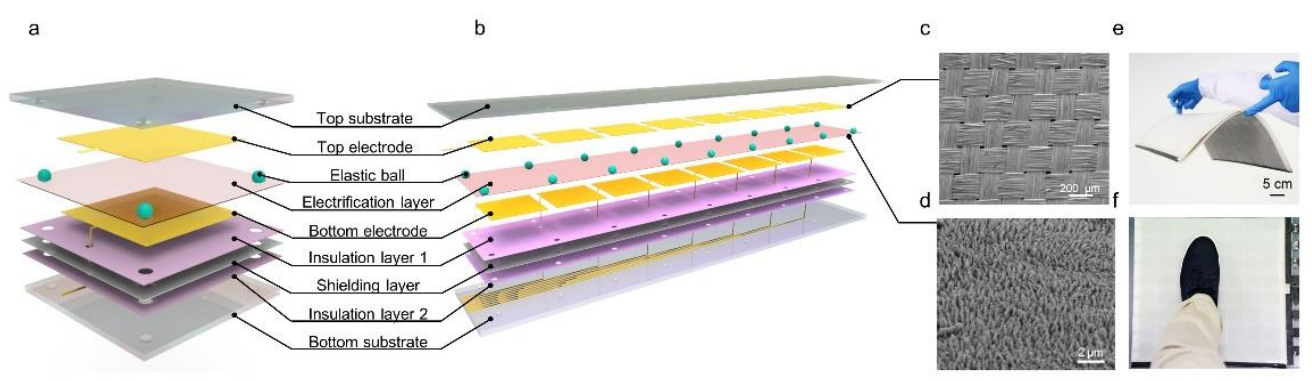

Figure 1. The structure and photographs of the TSA. Break-down structure of a) the single sensor unit b) a row of sensor units. c) SEM image of the top electrode. d) SEM image of the PTFE film. e-f) Pictures of the as-fabricated TSA.
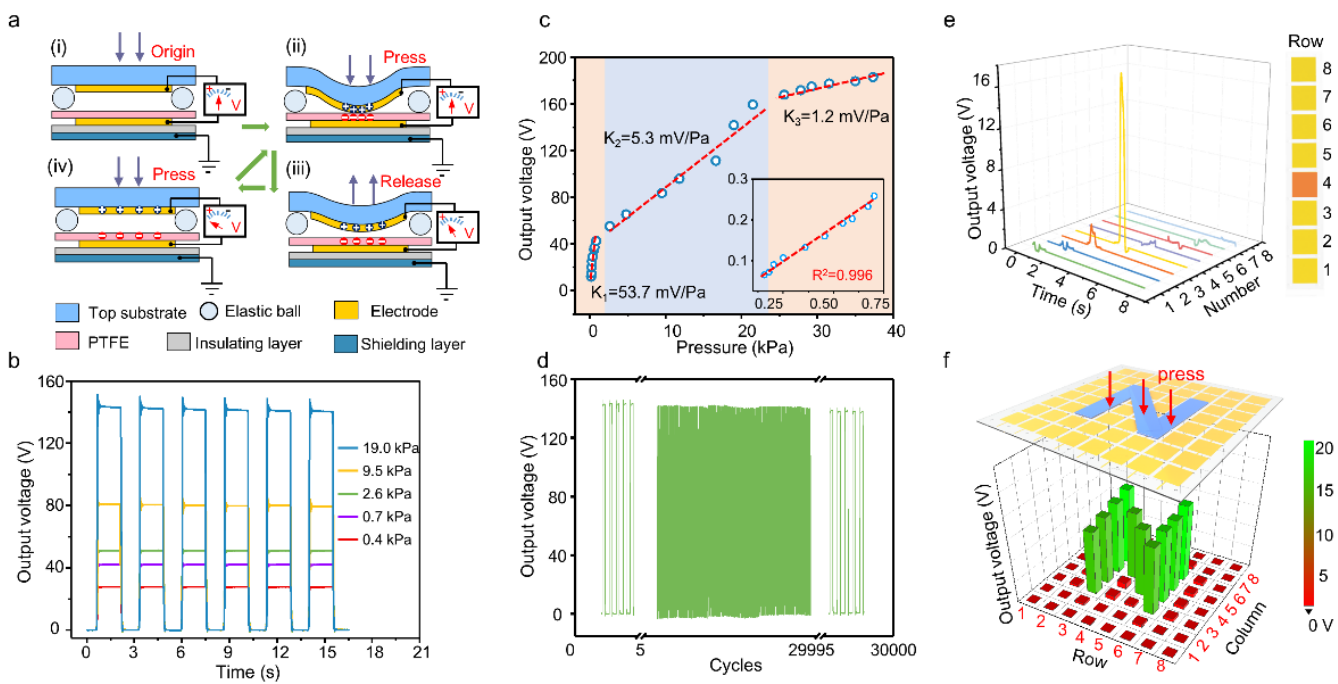

Figure 2. The working principle and performance characterization of the TSA. a) The working principle of the TSA. b) Output voltage of a sensor unit (with area of $4 \times 4 \mathrm{~cm}^{2}$ ) 
under different pressure. c) The sensibility curve of output voltage versus external pressure with pressure ranges from $0.1 \mathrm{kPa}$ to $37.5 \mathrm{kPa}$. d) Durability test with a single sensor unit. e) The output voltage of unit 4 when unit 1 to unit 8 were pressed successively with the presence of shielding layer. f) The output voltage image of the TSA when a model of shape "N" was pressed on the top surface.
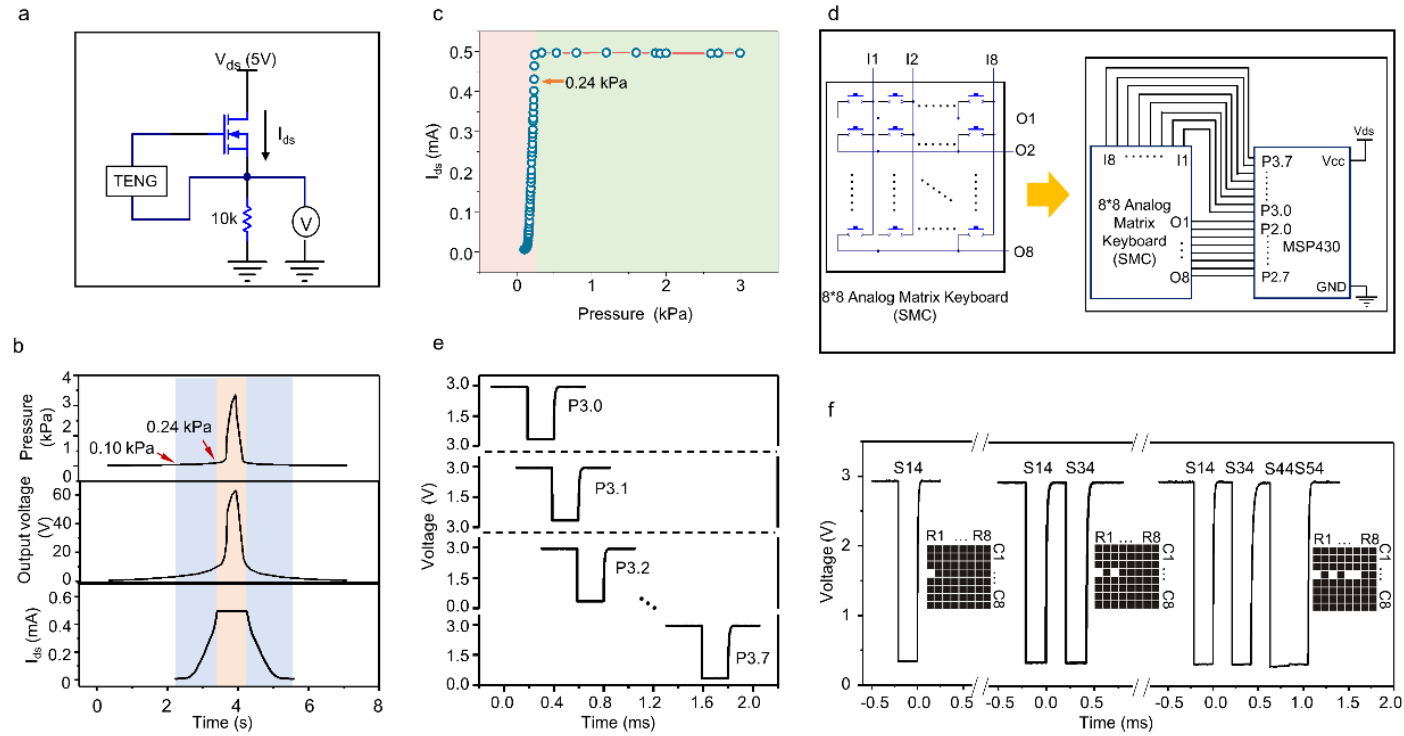

Figure 3. The fabrication and working mechanism of the ITSA. a) Circuit diagram of the combination way of one sensor unit in the TSA with a MOSFET. b) Measured pressure, output voltage and $I_{\mathrm{DS}}$ versus time with the hybrid TENG-MOSFET device. c) The sensibility curve of $I_{\text {DS }}$ versus pressure. d) Left: The equivalent circuit diagram of CD4066 array chip with eight input lines I1 to I8 and eight output lines O1 to O8. Right: The junction circuit between array chip of CD4066 and MCU. e) Low level signals of pin P3.0 to P3.7 in MCU. f) The working principle for positioning of the system. Left: single point test. Medium: two points test. Right: four points test. 
a
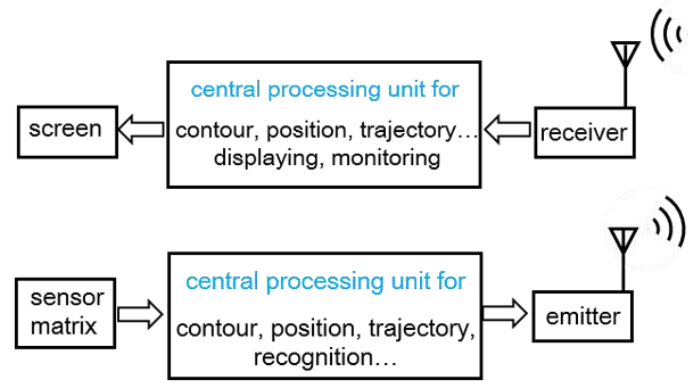

b

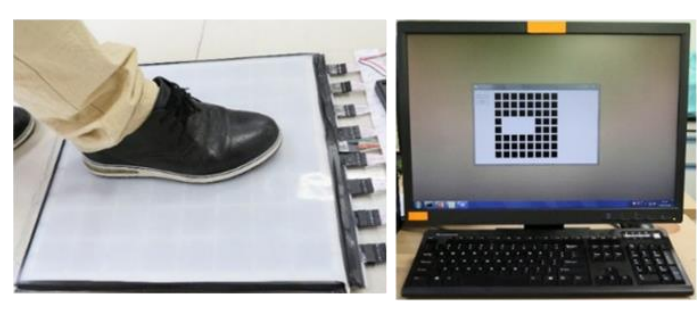

e

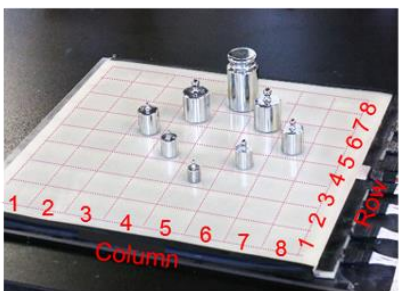

e
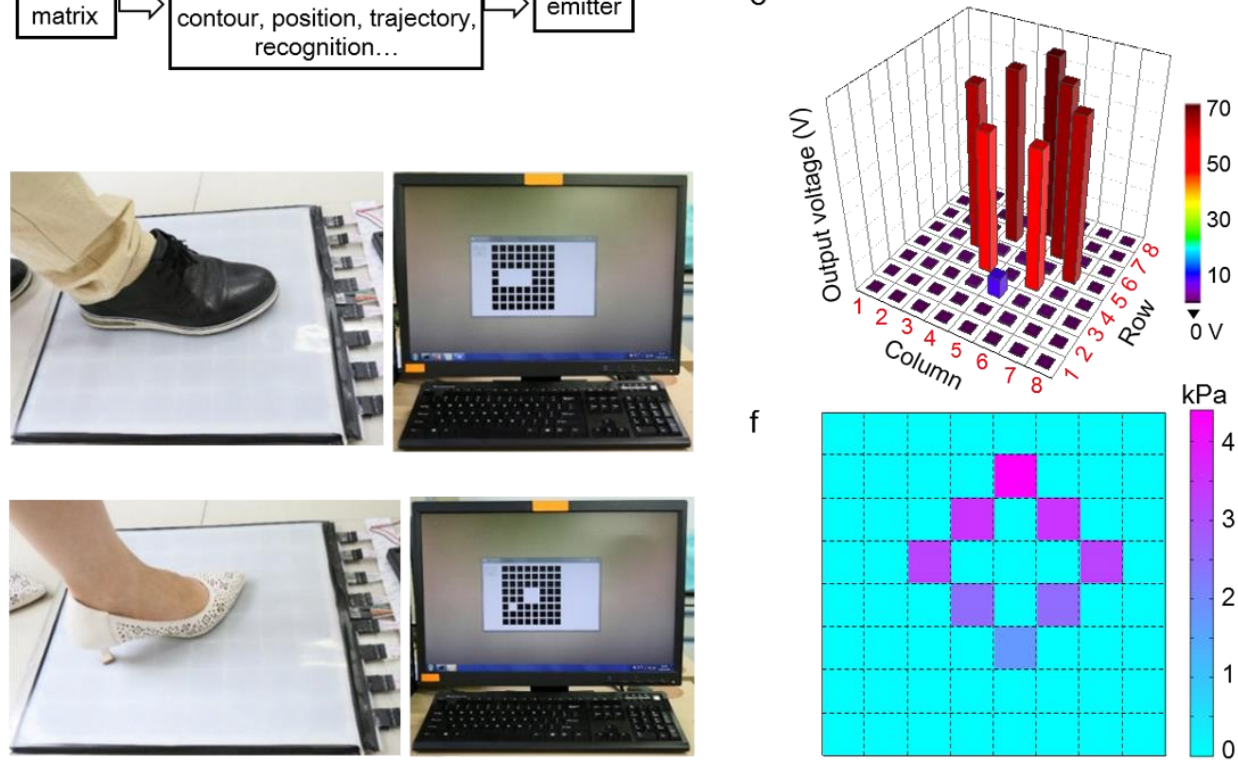

Figure 4. Practical wireless demonstrations of pressure sensing and mapping. a) The mechanism of visual and wireless pressure sensing. b-c) Wireless foot stepping experiments conducted with a leather shoe and a heel. d) The ITSA with counterweights distributed on the top. e) Reconstructed map with columns labelled with the counterweight identities and with their heights corresponding to the calibrated weights. f) Reconstructed map corresponding to the equated pressure of counterweights. 